\title{
Experiences with tungsten coatings in high heat flux tests and under plasma load in ASDEX Upgrade
}

\author{
A. Herrmann*, H. Greuner, J.C. Fuchs, P. de Marné, R. Neu and ASDEX Upgrade team \\ Max-Planck-Institut für Plasmaphysik, Euratom Association, 85748 Garching, Germany
}

\section{Abstract}

ASDEX Upgrade was operated with about $6400 \mathrm{~s}$ plasma discharge during the scientific program in $2007 / 2008$ exploring tungsten as first wall material in tokamaks. In a first phase the heating power was restricted to $10 \mathrm{MW}$. It was increased to $15 \mathrm{MW}$ in the second phase. During this operational period a delamination of the $200 \mu \mathrm{m}$ W-VPS coating at 2 out of 128 tiles of the outer divertor happens and an unscheduled opening was required. In a third phase ASDEX Upgrade was operated with partly predamaged tiles and up to $15 \mathrm{MW}$ heating power. The target load was actively controlled by $\mathrm{N}_{2}$-seeding. This paper presents the screening test of target tiles in the high heat flux test facility GLADIS, experience with operation and detected damages of the outer divertor as well as the heat load to the outer divertor and the reasons for the toroidal asymmetry of the divertor load.

PACS: 52.55.Rk, 61.30.Hn, 44.30.+v

*Corresponding author: Tel.: +49 8932991388; fax: +49 8932992580.

E-mail address: Albrecht.Herrmann@ipp.mpg.de.

\section{Introduction}

ASDEX Upgrade came into operation as divertor tokamak with a carbon first wall (FW) in 1991. During the last decade, it was changed stepwise to a tokamak experiment with a tungsten covered FW. In 2007 all FW components including the lower and upper divertor were made from tungsten coated fine grain graphite. ASDEX Upgrade became the first tokamak experiment with a tungsten FW [1]. 
The thickness of the tungsten coating was adjusted to the expected erosion due to plasma wall interaction. The outer strike line area was coated with $200 \mu \mathrm{m}$ thick vacuum plasma sprayed (VPS) tungsten, the inner strike line module as well as the upper divertor and the main chamber components has been coated with $4 \mu \mathrm{m}$ thick PVD tungsten (physical vapour deposition). The coatings were qualified by high heat flux screening and cyclic loading tests in the neutral beam facility GLADIS. About 3500 tungsten coated carbon tiles were installed in ASDEX Upgrade thereof 128 tiles with $200 \mu \mathrm{m}$ W-VPS coating at the lower outer strike line module.

During the scientific program in 2007/2008 about 1260 H-mode discharges with $P_{\text {heat }} \geq 5 M W$ were performed. From these H-mode discharges about $20 \%$ were high power discharges with $P_{\text {heat }} \geq 10 \mathrm{MW}$. During a phase with higher heating power 2 out of 128 tiles of the outer divertor suffered from a delamination of the W-VPS coating resulting accidentally in disruptions. The replacement of these tiles required an unscheduled opening. In summary, ASDEX Upgrade was successfully operated with H-mode discharges for more than 1600 shots or about $6400 \mathrm{~s}$.

This paper presents in the next section the results of the target screening in the high heat flux test facility GLADIS. Experiences of operation with W-VPS are presented in section 3. Finally, the failure scenario and the toroidal heat load variation due to geometric effects are presented in section 4 .

\section{Tile qualification in GLADIS}

The Garching Large Divertor Sample Test Facility (GLADIS) was designed as high heat flux test bed to qualify large plasma facing components [2,3]. The facility is equipped with two individual 1.1 MW RF-Ion sources. Each ion source delivers center heat flux densities between 3 and $45 \mathrm{MW} / \mathrm{m}^{2}$ with a beam diameter of $70 \mathrm{~mm}$ at the target position. Neutral 
beams, in contrast to electron beam heating, generate homogeneous heating due to the complete absorption of the beam power on the surface of the tested material. They are especially suited for loading of metallic surfaces, e.g. tungsten, where electron beam facilities loose up to $50 \%$ of the beam power due to reflection and formation of secondary electrons.

GLADIS was used to qualify tungsten coatings during the $R \& D$ process of coating technologies of fine grain graphite (FGG) and carbon fibre composite (CFC) based materials [4]. As a result FGG (R 6710) was selected as base material for the coating, because of the grain size and the thermal expansion coefficient that is nearest to that of tungsten, reducing the stress between the bulk material and the coating during the heating of the target. The use of CFC as base material was considered, in particular for high heat load components such as limiter and strike line tiles of the lower divertor but not realized due to the increased failure rate resulting from the inhomogeneous structure compared to FGG. In addition, FGG fits well to the requirements of ASDEX Upgrade discharges with typical up to $10 \mathrm{MW} / \mathrm{m}^{2}$ for about $3 \mathrm{~s}$.

The quality of the tungsten coating was monitored during the manufacturing by REM analysis of a control probe and layer characterisation (specific density, thickness from a microsection). In addition, the heat removal capability of selected tiles was screened in GLADIS before they were installed in ASDEX Upgrade. The typical screening procedure consists of:

Cyclic loading with $10.5 \mathrm{MW} / \mathrm{m}^{2}$ for $3.5 \mathrm{~s}$ with a cycle number between 10 and 200, representing the 'typical' ASDEX Upgrade divertor load for high power ( $\left.\mathrm{P}_{\text {heat }}=10-15 \mathrm{MW}\right)$ discharges.

Single pulses with an increased target load of about $23 \mathrm{MW} / \mathrm{m}^{2}$ for $1 \mathrm{~s}$ resulting in about the same surface temperature of about $1650^{\circ} \mathrm{C}$ as for the cyclic tests but with a higher temperature gradient into the coating and the bulk. 
During the tests the probe temperature is monitored routinely by a LWIR-camera and a bicolor pyrometer. A typical temperature pattern during heat loading of a target and the evolution of the maximum surface temperature for a cycle is shown in Fig. 1. After the tests the targets were inspected visually for cracks and melting. The temperature pattern reveals the Gaussian distribution of beam heat load. The temperature evolution shows an increased temperature during the first pulse(s) and a more or less constant temperature for the subsequent pulses. The higher temperature at the start of the cycling is due to dust and thermally weakly bound particles at the surface. These components are removed during the first pulses and afterwards the surface temperature keeps constant as long as the coating is not damaged.

\section{Tungsten coatings - experiences with operation}

The scientific program in 2007/2008 exploring tungsten as FW material in tokamaks had three phases. During a first phase the heating power was restricted to $10 \mathrm{MW} .476$ shots useful for the physical program were performed. Inspection of the in-vessel components during the scheduled opening in autumn 2007 reveals an excellent state of the FW components. The strike line targets were free of damages. Small damages were observed at the outer corners of the roof baffle. The second phase in 2008 was used to extend the operational space to plasma shots with higher plasma current $(<=1.2 \mathrm{MA})$ and higher heating power (up to $15 \mathrm{MW}$ ) resulting in a significant higher divertor load. For most of the discharges this caused no problem for the outer divertor, which is the component with the highest power and energy deposition despite the fact that the divertor surface temperature exceeded $1000^{\circ} \mathrm{C}$. A few discharges show an increase of the divertor heat load for otherwise the same core plasma parameters to a maximum heat load considerably above $10 \mathrm{MW} / \mathrm{m}^{2}[5]$. As a consequence, the thick tungsten coating at the outer strike line delaminated at 2 out of 128 tiles, limiting regular plasma operation because large delaminating tungsten flakes 
resulted in disruptions. Fig. 2 shows the lower outer divertor with a delaminated layer at one tile. It should be mentioned here that the delamination did not start at the edge of the target tile but is over the whole target area. This delamination required a maintenance opening to exchange the damaged target tiles. After a similar subsequent failure of the coating it was possible to continue with operation for several weeks until the scheduled summer opening. The in-vessel inspection revealed that the outer strike point tiles suffers from different damages -6 tiles show a partial delamination of the thick layer, 10 tiles suffered from melting of sub-layers of the $200 \mu \mathrm{m}$ W-VPS coating with droplets in the molten region, 18 tiles show cracks going through the $200 \mu \mathrm{m}$ coating. One of these 'predamaged' tiles was screened in GLADIS. No further damages were observed during the cyclic loading by 10.5 $\mathrm{MW} / \mathrm{m}^{2}(3.5 \mathrm{~s})$. First delaminations occur after the third pulse at the highest heat load (23 $\mathrm{MW} / \mathrm{m}^{2}$ for $\left.1 \mathrm{~s}\right)$.

The delaminated tiles were replaced by tiles with a $10 \mu \mathrm{m}$ CMSII-tungsten coating [6]. Tiles with cracks and melting zones with a small area were not replaced due to a lack of spare parts. ASDEX Upgrade was operated with this configuration in a third phase for another 500 shots and up to $15 \mathrm{MW}$ heating power. The divertor was protected against overload passively by a video real time control and actively by the implementation of nitrogen seeding for better energy redistribution in the divertor.

\section{Failure scenario and divertor geometry}

The partial delamination of the W-VPS layer appeared after running a shot after boronization with a heating power of $12.5 \mathrm{MW}$. A comparable shot was run before boronization without any consequences for the divertor. Fig. 3 shows a video image from the top onto the lower divertor of the two comparable shots. It is obvious from the brightness of the divertor tiles that the discharge after boronization results in a significantly higher divertor load. It is also 
obvious from Fig. 3 that there is a toroidal variation of the target temperature, i.e. the heat load to the target. The power crossing the separatrix calculated from global plasma parameters, $\mathrm{P}_{\text {heat }}$ and $\mathrm{P}_{\text {radiated }}$ related to the same wetted area $\left(\mathrm{A}_{\text {wetted }}\right)$ for both discharges $q_{\text {sep }}=\frac{P_{\text {heat }}-P_{\text {rad }}}{A_{\text {wetted }}}$ as plotted in Fig. 4, shows comparable temporal behaviour and similar absolute values. This is in contrast to the real temperature as measured by the fast standard thermography. The latter is about a factor of 2 higher for the discharge with boronization, i.e. the same global plasma parameters result in significantly different divertor parameters. The time averaged $(50 \mathrm{~ms})$ heat load calculated from the temperature evolution is $7 \mathrm{MW} / \mathrm{m}^{2}$ and $15 \mathrm{MW} / \mathrm{m}^{2}$, respectively, at $4 \mathrm{~s}$ discharge time [5].

The fast standard thermography is measuring at only one toroidal position so that the indicated temperature did not necessarily represent the location with the maximum temperature or the temperature of the damaged target tiles. In particular, because of the toroidal variation of the temperature as shown in Fig. 3.

The toroidal variation of the divertor load has geometric reasons which originate from the divertor design and alignment. The ASDEX Upgrade outer divertor consists of 16 support structures, each carrying 8 target tiles with a flat surface, i.e. the major radius of the outer divertor of $1.6 \mathrm{~m}$ is approximated by a polygon with 128 edges. The deviation of a target tile from a circle results in a small variation of the major radius of $+-0.4 \mathrm{~mm}$.

Moreover, the tiles are grouped as double packages. There is no gap inside such a package but tungsten coating requires rounded edges. That's why the second tile is shadowed with a shadow depth of $0.4 \mathrm{~mm}$. The double packages are separated by $4 \mathrm{~mm}$ wide gaps that are used for diagnostics views. To avoid edge effects, the first tile of a double package is shadowed with a shadow depth of $0.8 \mathrm{~mm}$. The applied target tilting is $1.7^{\circ}$ and $2.1^{\circ}$, respectively. The difference of $0.4^{\circ}$ has to be compared to the total tilting angle, the sum of the geometric tilting 
angle and the magnetic pitch angle that is between $2^{\circ}$ and $4^{\circ}$ depending on the magnetic configuration (safety factor). From the different target tilting a spatial variation of the temperature below $10 \%$ is expected. The temperature variation across a single tile is caused by the polygonial shape of the divertor and the resulting variation of the angle between the nominal radius and the target of $\pm 1.4^{\circ}$. A third factor causing toroidal variations of the heat load is the adjustment of the support structure that can modify the effective pitch angle for 8 tiles as a group. The consequences of these effects are illustrated in Fig. 5.

\section{Summary}

ASDEX Upgrade was operated with about $6400 \mathrm{~s}$ plasma discharge during the scientific program in $2007 / 2008$ exploring tungsten as FW material in tokamaks. In a first phase the heating power was restricted to $10 \mathrm{MW}$. It was increased to $15 \mathrm{MW}$ in second phase. During this operational period a delamination of the $200 \mu \mathrm{m}$ W-VPS coating at the outer lower divertor occurs requiring an unscheduled opening for repair. In a third phase ASDEX Upgrade was operated with up to $15 \mathrm{MW}$ heating power and a predamaged outer divertor. Active divertor cooling by $\mathrm{N}_{2}$-seeding and video-real-time control of the divertor brightness ensures save operation.

The heat removal capability of selected target tiles installed in ASDEX Upgrade was screened at the IPP site in GLADIS. Typical screening parameters were 10-200 pulses with 10.5 $\mathrm{MW} / \mathrm{m}^{2}$ for $3.5 \mathrm{~s}$ and single pulses with $23 \mathrm{MW} / \mathrm{m}^{2}$ for $1 \mathrm{~s}$. Target tiles tested before installation showed no damage. A target tile with a crack in the $\mathrm{W}$ coating taken out of the divertor after phase 2 of operation show no degradation in the cyclic test but fails after 3 pulses at $23 \mathrm{MW} / \mathrm{m}^{2}$.

Delamination of the W-VPS layer at 2 out of 128 tiles appeared after discharges after boronization with high divertor load. During this shot, the local heat load at the outer target 
exceeded the design heat load of $10.5 \mathrm{MW} / \mathrm{m}^{2}$ significantly. The toroidal variation of the heat load due to geometric effects enhances the danger of target overload.

Different levels of damages at the thick W-coating were observed during routine in-vessel inspection. 6 tiles suffer from a delamination of the thick W-coating. This type of damage caused problems during plasma operation. A few tiles show a melting of the top layer(s) with droplets and a significant number of tiles reveal cracks through the thick layer. These cracks did not limit the save operation with the standard parameters as verified with GLADIS.

In summary, ASDEX Upgrade could be successfully operated with a tungsten FW. A limitation for plasma operation due to a delamination of the thick W-coating was caused by discharges reaching or exceeding the 'typical' divertor load of $10.5 \mathrm{MW} / \mathrm{m}^{2}$ for longer time periods.

\section{References}

[1] Neu R et al 2007 Plasma Phys. Control. Fusion 49 B59-B70

[2] Greuner $\mathrm{H}$ et al 2005 Fusion Eng. Des. 75-9 345-350

[3] Greuner H et al 2007 J. Nucl. Mater. 367-370 1444-1448

[4] Neu R et al 2007 Phys. Scrip. 2007150.

[5] Herrmann A et al. 2009 of Conference, "Change from low to high divertor load and edge profiles in comparable $\mathrm{H}$-mode discharges in ASDEX Upgrade"; Proc. of the 36th EPS Conference on Controlled Fusion and Plasma Physics, Sofia 2009.

[6] Ruset C et al 2007 Phys. Scrip. T128 171-174 


\section{Figures}

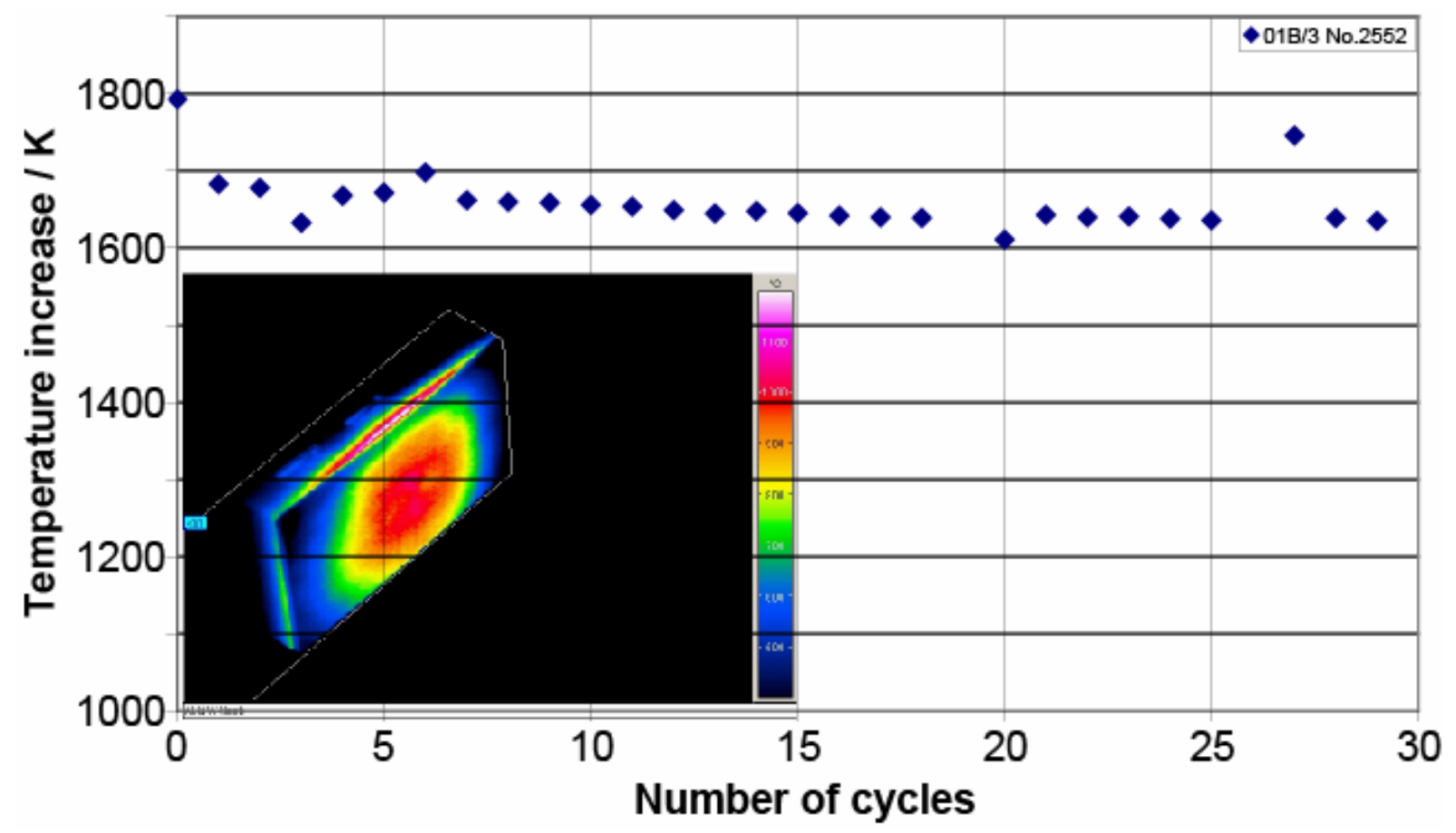

Fig. 1 Cyclic load test of an AUG tungsten coated (W-VPS $200 \mu \mathrm{m}$ ) target tile. Temperature increase vs. cycle number for a heat load of $10.5 \mathrm{MW} / \mathrm{m}^{2}$ for $3.5 \mathrm{~s}$. Insert: Typical IR image of a target tile at the end of a cycle.

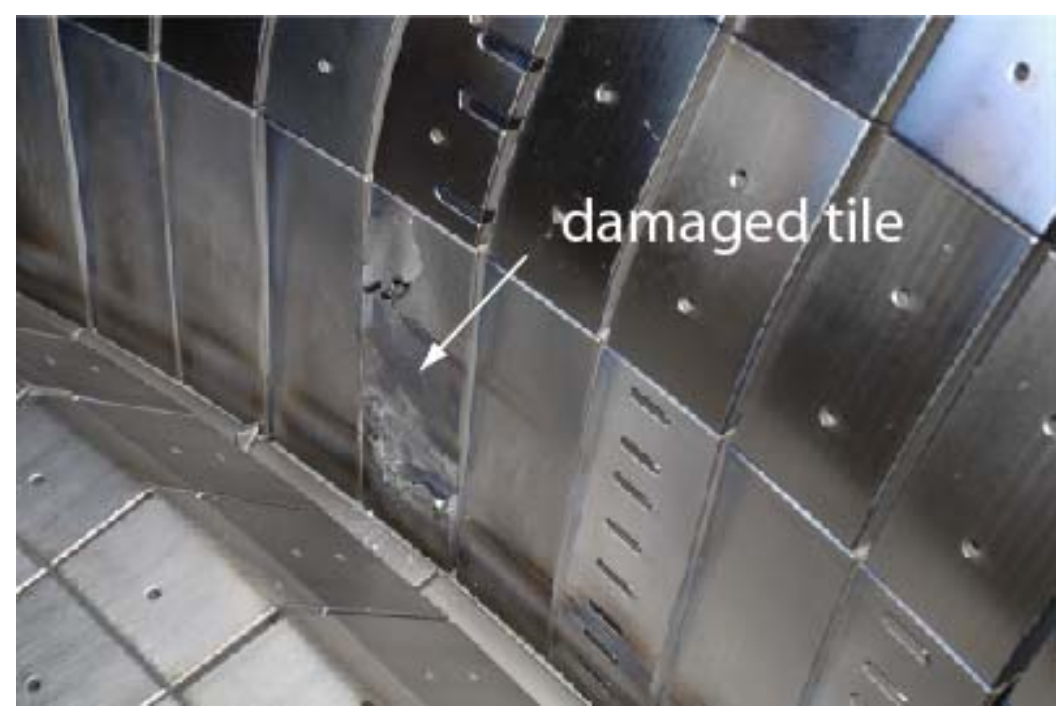

Fig. 2 Delaminated $200 \mu \mathrm{m}$ tungsten coating in the lower outer divertor. 

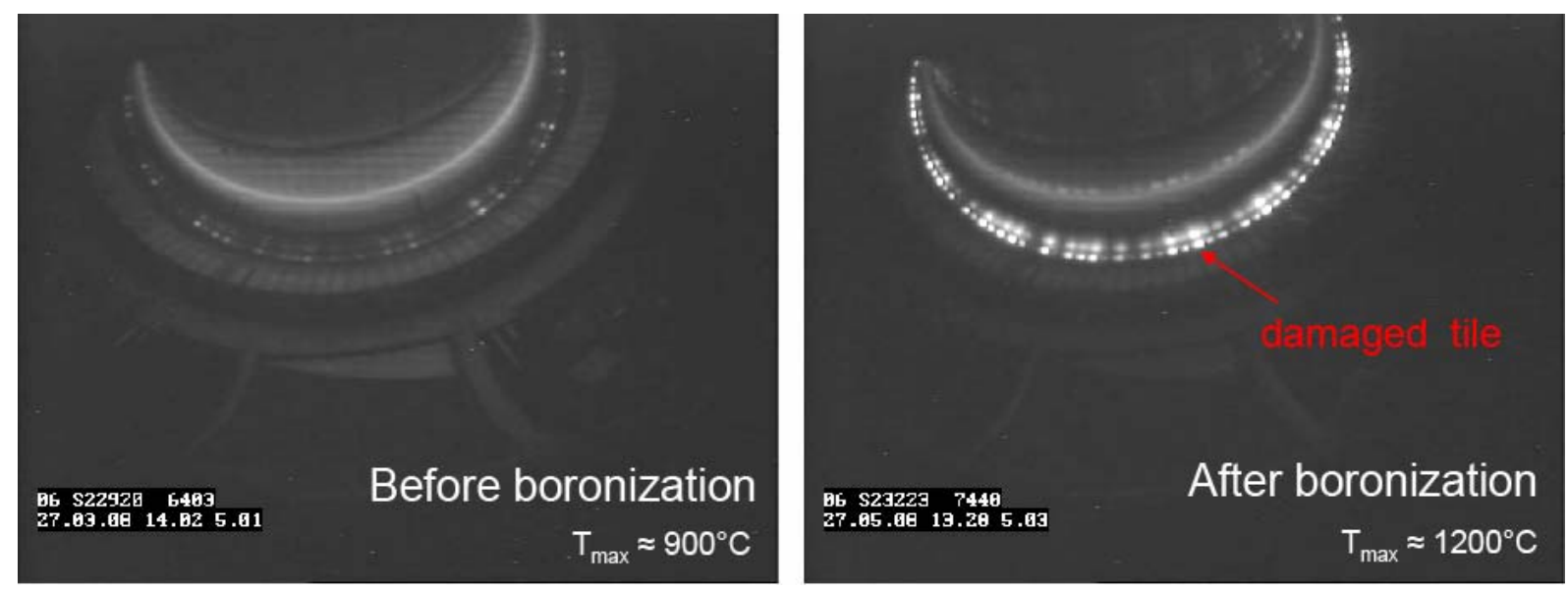

Fig. 3 Video image of the lower divertor for 2 comparable discharges with and w/o boronization.

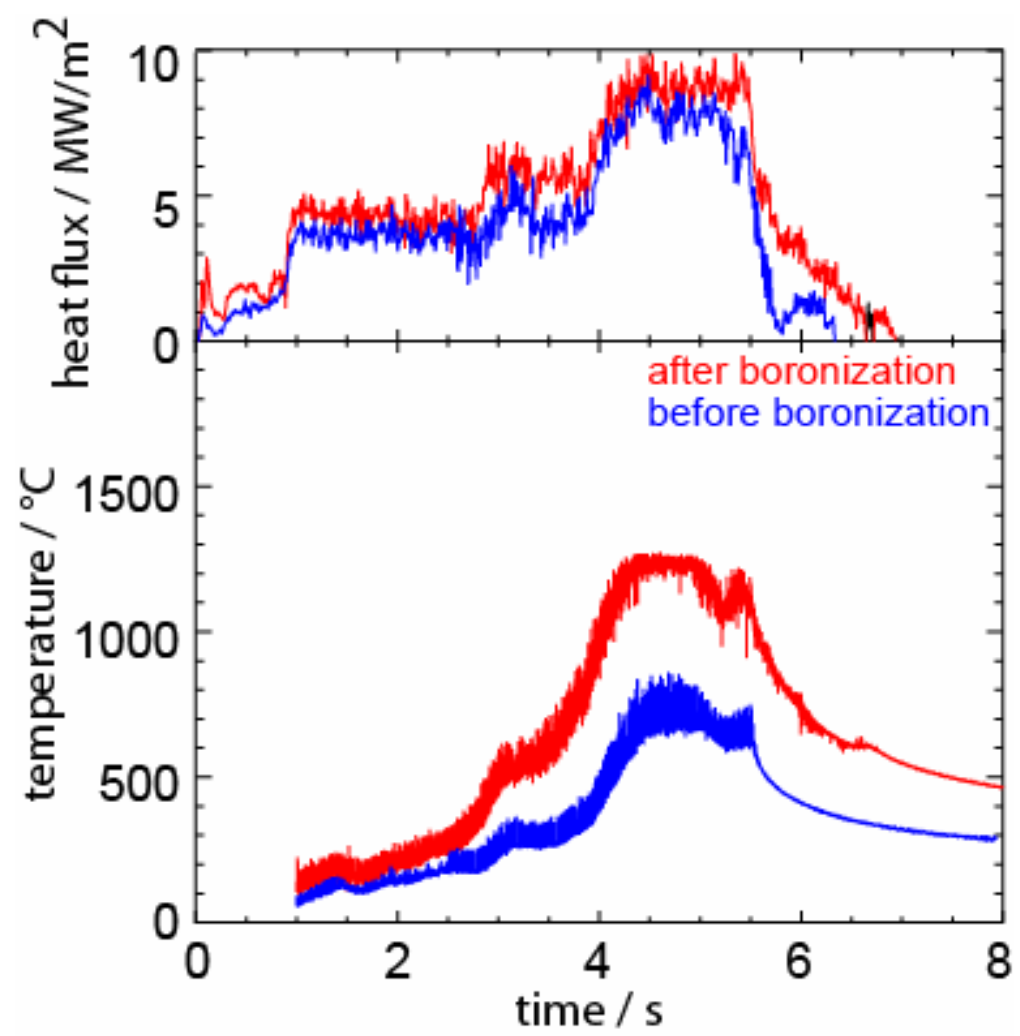

Fig. 4 Heat flux across the separatrix, calculated from core plasma parameters $\left(\left(P_{\text {heat }}-P_{\text {rad }}\right) / A_{\text {wetted }}\right)$ for 2 discharges and the measured maximum surface temperature at 1 toroidal location in the lower outer divertor. The temperature measurement is saturated at about $1300^{\circ} \mathrm{C}$. 


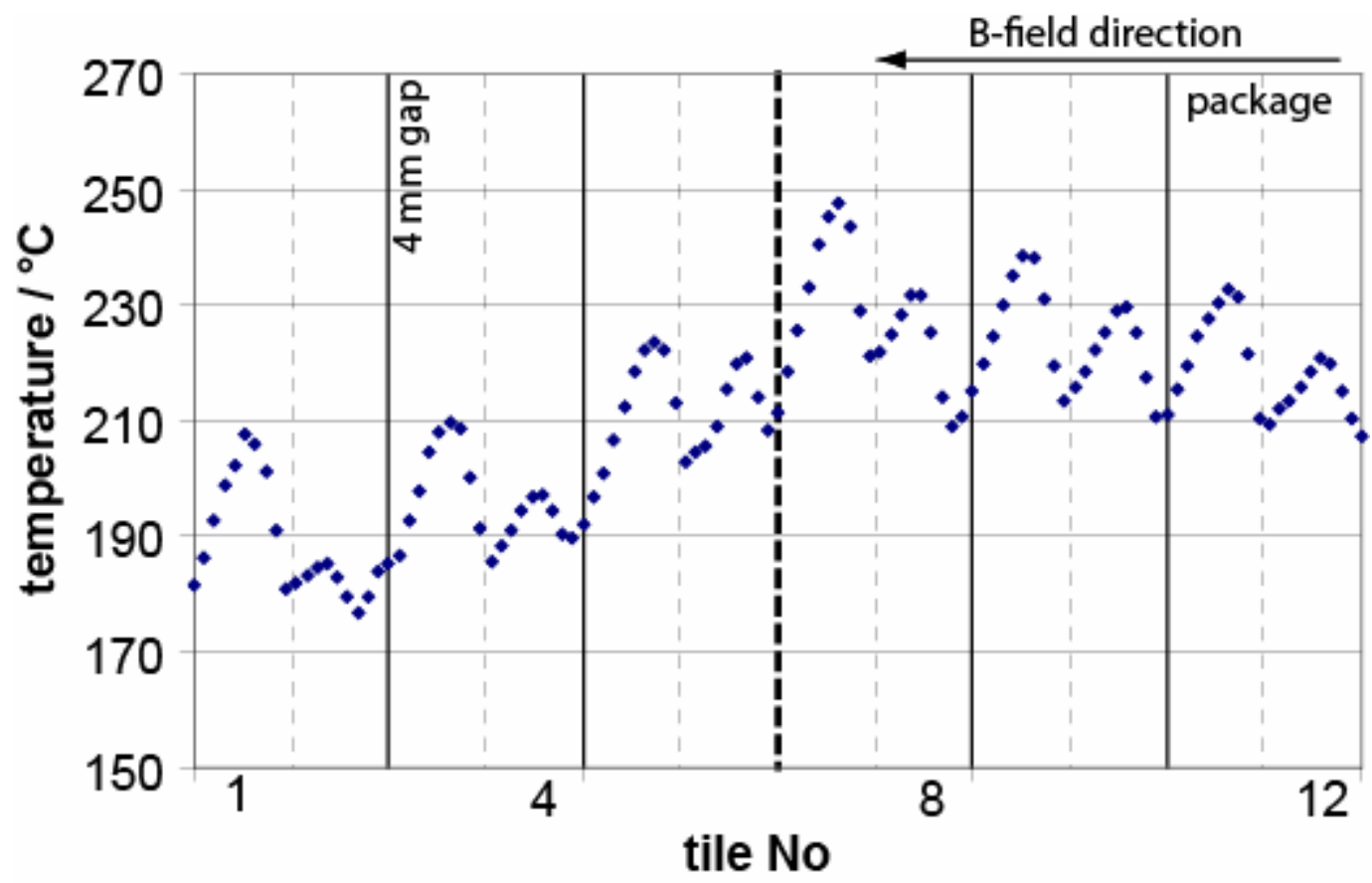

Fig. 5 Surface temperature at the outer strike line as measured with a 2D IR camera viewing tangential into the lower divertor. The thick dashed line marks the change between support structures. The thick lines indicate double packages, i.e. the $4 \mathrm{~mm}$ gap. Data are taken at the beginning of the 5MW phase of an H-mode discharge (23429 @ 2s). 